\title{
GIROSCOPIE DE LA CRISE
}

\author{
Author(s) / Auteur(s) : \\ Andrée PIECQ \\ Secrétaire générale de l'Union européenne de systémique (UES) \\ Directrice scientifique de l'Institut Indépendant de Systémique des organisations (G.I.R.O.S) \\ Présidente d'honneur de l'asbl Systèmes \& Organisations $(S \& O)$ \\ a.piecq@gmail.com
}

\section{Abstract / Résumé :}

L'objectif de cet article est d'analyser, avec un regard systémique, les crises, qui bien que de durées différentes, ont ponctué ces dernières décennies. Elles ont émergé et émergent dans presque tous les secteurs d'activités tel que en politique, en économique, en écologique, dans la culture, dans les rapports sociaux, dans l'enseignement...

La méthodologie utilisée pour cette analyse est celle du "Giroscope » et de ses douze "Principes Directeurs des Systèmes », un modèle d'application des concepts systémiques.

- Dans le premier temps «l'aspect étymologique» de la crise est abordé, suivi de quelques exemples dans le domaine courant.

- Dans un second temps les indices de la crise vont être décelés à l'aide de «Principes Directeurs »du «Giroscope» et des contextes de la crise.

1. La finalité

2. Les frontières

3. Les règles,

4. Les rétroactions,

5. L'homéostasie,

6. L'équifinalité

7. L'émission, réception de l'information.

- Dans un troisième temps les types de changement à effectuer ou à fuir vont être définis.

La crise du 2le siècle ne serait-elle pas la non crise? Une question à se poser face à l'inflation des crises de tout ordre.

\section{Keywords / Mots-clés :}

crises, systèmes, complexité, méta crise, changement

\section{PRÉALABLE}

Depuis plusieurs siècles le mot crise est utilisé lorsque quelque chose va mal, lorsqu'une situation devient difficile à gérer. Depuis la fin du $20^{\mathrm{e}}$ siècle, si l'on en croit les médias, c'est comme si il y avait une inflation de crises car elles n'arrêtent pas de se succéder. L'observation montre qu'elles touchent tous les secteurs que ce soit en politique, en l'économie, en l'écologie, dans la culture, dans les rapports sociaux, dans l'enseignement, dans l'installation de l'identité qui frappe aujourd'hui tous les pays du fait de l'afflux des réfugiés tant chez les autochtones que chez les réfugiés. Bref elle touche toute la société.

- Le mot crise se retrouve à l'origine dans le mot grec «krisis » dont la racine indoeuropéenne est « krei » qui signifie juger, distinguer, trier des éléments qui se mélanges.

- En grec le mot crise Kairos ${ }^{1}$ à le sens de jugement, de décision efficace entre deux choix possibles qui survient après leur évaluation et nécessite une action à un moment opportun.

\footnotetext{
${ }^{1}$ Centre de Recherches Interdisciplinaires en Sciences Humaines et Sociales de Montpellier (C. R. I. S. e. S)
} 
- En latin la crise est associée à la manifestation brutale d'une maladie, c'est l'instant ou la maladie explose, ou elle culmine.

- En utilisant le «giroscope» la crise peut être considérée comme si elle est un «système espace/action » dans lequel des finalités opposées s'affrontent pour conduire le système vers le changement en modifiant sa structure.

Dans toutes ces «définitions » de la crise, ce qui est redondant c'est que toutes comportent des verbes d'action,

Même en chinois qui a une racine totalement différente, le mot crise est représenté par deux idéogrammes dont le sens est à la fois danger et opportunité qui porte en eux également de l'action.

L'observation de l'histoire montre qu'elles n'arrivent pas par hasard. Elles s'inscrivent toute dans un contexte et sont les prémisses d'un changement

- Sont-elles une opportunité, une nécessité, un plus dans l'évolution?

- Sont-elles le symptôme que le système est en danger et qu'il doit changer?

- Sont-elles un détecteur d'incendie?

- Des moments sans crises ont-ils apparu dans l'histoire ?

- Un système sans crise est-il fonctionnel ?

- Mettent-elles en péril les objectifs et de là, la survie des sociétés ?

- Pour sortir de la crise les symptômes observés doivent ils atteindre un état paroxystique ou la seule solution de survie est le changement?

- De quel ordre est ce changement?

- Sont-elles le moment charnière où le système bascule du non changement, de l'homéostasie vers le changement, de la mort à la vie ?

Toutes les crises sont-elles isomorphes?.

\section{« GIROSCOPIE»² DE LA CRISE}

\section{Introduction}

Le système crise est un système « espace/action » qui se compose de sous-systèmes dont les finalités ${ }^{3}$ ne sont plus dans des rapports complémentaires opérationnels. En effet, suite à des modifications contextuelles avec lequel le système est en interaction, ces rapports se rigidifient et s'opposent, de l'instabilité s'installe. C'est comme si le système était pris dans une situation paradoxale et ne pouvait "choisir" entre ses finalités opposées. Après leur évaluation une action faite « au moment opportun ${ }^{4}$ », modifiera les rapports entre ses finalités qui deviennent alors symétriques, s'entrechoquent et conduisent à l'explosion.

Pour illustrer ces propose et répondre aux questions posées le système «crise espace/action » va être regardé à travers différentes crises qui ont secouées le monde.

\section{Les contextes des systèmes « espace/action crise »}

L'observation des contextes dans lesquels ces systèmes s'inscrivent montrent l'importance :

\footnotetext{
${ }^{2}$ Utilisation $\mathrm{du}$ « giroscope » une méthodologie pour diagnostiquer les systèmes, les organisations.systémique voir « De la systémique à la pratique de l'Organisations - le « giroscope » Andrée Piecq

${ }^{3}$ Les finalités sont une émergence d'une vision du système qui pour être opérationnelle doit être définie dans le temps et se décliner en trois niveaux interdépendant : dans le long terme en buts à atteindre ; dans le moyen terme en objectifs et dans le court terme en actions nécessaires à la réussite de ces objectifs.

${ }^{4}$ Voir la définition de Kairos
} 


\section{Des contextes économiques et sociaux}

En 1788 en France les récoltes sont maigres ce qui conduit à une diminution des ressources et à une augmentation de la pauvreté ; les ordres privilégiés (le clergé et la noblesse) ne paient pas d'impôts ; la fiscalité est lourde pour le Tiers Etat qui représente la plus grande partie de la population ${ }^{5}$. Le budget de l'État est en énorme déficit.

En Mai 68, des usines ferment, il y a 500.000 chômeurs, il y a 5.000 .000 de pauvres. Un mouvement de contestation part de l'université de Nanterre le 22 mars et se communique au monde ouvrier.

En 1960-1961 en Belgique « la grève du siècle » émergent dans un contexte de crise économique suite à la perte du Congo et d'une décennie d'agitation sociale.

La crise financière mondiale de 2007-2008 émerge au Etats-Unis de la possibilité que des emprunteurs à risques puissent faire des crédits hypothécaires rechargeables ${ }^{6}$ et à taux variables. La banque Centrale augmente ses taux, il y a une baisse du prix de l'immobilier. Les établissements financiers subissent de lourdes pertes. Il y a une chute des marchés boursiers.

\section{Des contextes politiques}

En 1788 la monarchie de droit divin est affaiblie. Le roi gouverne avec de simple technicien ce qui conduit à un blocage politique. La structure de la société est remise en question.

En mai 68 la République gaullienne, en place depuis 1958 subit une usure du pouvoir. La jeunesse manifeste contre la guerre du Vietnam, les dysfonctionnements de l'enseignement supérieur et la société de consommation. Cette manifestation s'inscrit dans une crise internationale qui a pris naissance aux États-Unis : en septembre 1964, sur le campus de Berkeley, le Free Speech Mouvement lance la protestation contre la guerre du Vietnam.

En 1960-61, le Gouvernement Eyskens décide d'une politique d'austérité, qui consistait en une augmentation de la fiscalité, d'une diminution des pensions de retraite du secteur public...; des mesures impopulaires réunies sous l'appellation Loi Unique.

La crise financière de 2007-2008 s'inscrit dans des politiques économiques basées sur l'accroissement de l'endettement de l'Etat.

\section{Des contextes philosophiques}

En 1788 les philosophes des lumières, Montesquieu, Diderot, Rousseau, et Voltaire ont influencé les pensées révolutionnaires de 1788. Les idées de Rousseau en faveur de la République (la souveraineté du peuple) et celle de Voltaire la défense la "liberté d'expression" étaient dangereuses pour la royauté.

En Mai 68 une philosophie hédoniste prônant l'épanouissement de l'individu, son droit à la libération de la parole et à la libération sexuelle s'inscrit dans une contestation de la société de consommation, de l'idéologie productiviste et de la dépendance aux besoins et aux nouveaux objets.

Lors des grèves de 1960-61 la cassure idéologique nord sud en Belgique se manifeste. La grève est moins forte dans le nord plus prospère.

La crise qui a secoué le monde en 2007-2008 est manifestement une crise du modèle : une société articulée sur la paire marché et démocratie (Attali, 2009)

\section{Les Finalités qui sont en présences}

L'observation montre que dans ces types de contextes de nouveaux sous-systèmes émergent avec des finalités différentes ou ceux qui existent ont leurs finalités changées.

Tout système a une finalité dans lequel les sous-systèmes s'insèrent avec des buts, des objectifs et des actions complémentaires nécessaire pour que le système soit opérationnel. Lorsque les finalités des sous-systèmes et des membres entrent en conflits ou en contradictions avec celles du système, le système rencontre des turbulences et s'interroge sur la pertinence de sa finalité. Il juge, distingue, trie les éléments qui se mélanges (cf. l'étymologie du mot crise). Si ce tri n'est pas possible, c'est comme

\footnotetext{
${ }^{5}$ Le tiers Etats est constitué des bourgeois, des artisans, des ouvriers et des paysans ;

${ }^{6}$ Crédits rechargeables : l'emprunteur peut se réendetter en fonction de l'augmentation de la valeur de son patrimoine.
} 
si le système ne pouvait pas changer qu'il est dans un état homéostatique, que les rapports entre les sous-systèmes sont complémentaires rigidifiés. Le système stagne dans cet état.

Des éléments contextuels vont pousser les sous-systèmes à se battre pour le «pouvoir », pour quitter cet état de stagnation. Les rapports entre sous-systèmes changent et leurs finalités deviennent symétriques et conduit jusqu'à la seule issue, l'explosion ou le système va changer de structure.

Dans les exemples ci-dessus nous nous pencherons plus particulièrement sur la France de 1788-1789.

Avant $1788 \mathrm{La}$ France était composée de sous-systèmes qui étaient dans des rapports complémentaires.

En 1788-1789 les sous-systèmes qui la composent sont :

- Les nobles ;

- Le clergé qui se divise en haut clergé aristocratique et en bas clergé provenant du peuple ;

- Le tiers Etats qui comprend la bourgeoisie (industriels, banquiers, commerçants, médecin, avocat, ...) les artisans ou les ouvriers et les paysans (le système dont les membres sont les plus nombreux).

Ces sous-systèmes ont des visions différentes de la société qui les conduisent à des finalités qui s'opposent :

- Au niveau des buts :

Les nobles et le haut clergé veulent la conservation, de leurs privilèges ; La bourgeoisie souhaite une réforme de la société et du système politique et une reconnaissance sociale qui transformerait ses membres en notables de plein droit ; Les buts du bas clergé, des artisans, des ouvriers et des paysans sont une amélioration des conditions de vie.

- Au niveau des objectifs : Les nobles et le haut clergé ont pour objectif l'opposition à l'évolution économique et sociale qui les marginalise.

Le bas clergé a pour objectifs la lutte contre l'aristocratique haut clergé ; l'accès aux hautes fonctions ecclésiastiques et l'abandon des privilèges fiscaux.

Les bourgeois ont pour objectifs de faire tomber les barrières qui empêchent l'économie et le développement de la production et d'avoir l'égalité juridique et fiscale

Les artisans, les ouvriers (le petit peuple) ont pour objectifs leur survie, une réponse à leurs besoins matériels tels que la fin de la surimposition, la diminution prix des denrées alimentaires.

Les paysans ont pour objectif la libération du joug féodal et la propriété des terres.

Ces finalités (buts et objectifs) sont devenues non seulement différentes mais incompatibles. Elles ne sont plus complémentaires mais symétriques elles sont en escalade (c'est à celle qui sera supérieur à l'autre) et les actions qui vont être nécessaires à leur réalisation sont également en symétrie. Cette incompatibilité, cette symétrie des finalités en présence va conduire à une explosion : la Révolution Française de 1788-1789.

L'hypothèse faite à partir de ces observations est comme si l'acmé de la crise émerge lorsque des inégalités sont trop fortes et que :

- Les frontières entre les sous-systèmes se rigidifient ;

- Les supports de la communication deviennent de plus en plus analogiques (prise de la bastille, décapitation du roi...) et définissent les relations entre les sous-systèmes comme symétriques ;

- Les sous-systèmes veulent être égaux et luttent pour le contrôle, la prise de pouvoir. Cette rivalité conduit à l'emballement du système qui entre en guerre jusqu'à la rupture totale et le rejet de l'autre ;

- Les membres, les individus qui composent ces sous-systèmes sont en souffrance, leur rôle et leur fonction sont soumises à des changements rapides ; 
- Les règles mythiques sont ébranlées car la vision du système a changé, les règles explicites sont en voie d'élaboration et de ce fait un grand nombre de règles sont de l'ordre de l'implicite.

Les rétroactions se font sur le mode de l'escalade et il n'y a plus de métacommunication possible ce qui conduit à des actions violentes Les rétroactions écartent le système de son point d'équilibre et le conduisent vers le changement ce qui permet l'évolution du système. Le désordre augmente et de ce désordre une nouvelle stabilité va apparaître.

\section{CONCLUSIONS EN REPONSE AUX QUESTIONS POSEES DANS LE PREALABLE}

L'histoire de l'apparition et de l'évolution de la planète terre ainsi que l'évolution des sociétés humaines permet de dire que c'est comme si elles faisaient suite à des modifications structurelles provenant du choc d'éléments dans le cas de la terre, (que l'on peut considérer comme des finalités différentes et opposées) et dans l'évolution des sociétés humaines comme le choc de finalités opposées.

L'observation du monde occidental depuis la fin de la guerre 1940 montre que si certains soussystèmes de ce monde ont pu être considérer sans crise, le système global ne l'a jamais été et ne le sera probablement jamais.

Un système sans crise existe et il est fonctionnel. Les finalités de ses sous-systèmes ne s'opposent pas et sont dans des rapports complémentaires. Ce type de système est opérant et les changements se font sans modification de la structure du système. Des aménagements à l'intérieur des principes directeurs ${ }^{7}$ suffisent car les sociétés fonctionnent et évolue.

L'histoire montre que lorsque ces sociétés peuvent ne plus évoluer, rester coincées et devenir des systèmes fermés dont les finalités ne sont plus adaptées aux contextes dans lesquelles elles se trouvent. Elles restent dans un état homéostatique sans changement possible. C'est alors que les crises surviennent, et lorsqu'elles atteignent un niveau paroxystique la société, système fermé, meurt pour revivre avec une autre structure. En d'autres mots la société a fait une mutation ${ }^{8}$ qui va modifier les rapports qui existe entre les principes directeurs (frontières, membres sous-systèmes, règles, finalités, totalité, rétroactions...

L'observation des systèmes en crise quel qu'ils soient (systèmes vivants ou non) permet de dire qu'ils sont isomorphes en effet leur structure présente toute des finalités opposées qui s'affrontent et les rapporte entre leurs principes directeurs sont équivalents.

En ce $21^{\mathrm{e}}$ siècle l'inflation des crises qui apparaissent dans tous les secteurs, n'a pas atteint un point de rupture qui permette le basculement d'une ancienne structure vers une nouvelle structure de la société. Selon le «Giroscope » pour conduire un système non opérant au changement il faut une mutation, or l'observation montre que la structure de la société ne change pas. Ce qui est fait ce sont des modifications des contenus traités dans les domaines en crise mais non des modifications de rapport entre les principes directeurs qui pourraient conduire à une mutation.

$\mathrm{Au}$ regard de l'histoire, cette mutation risque de se faire dans la violence entre états, ou individus ou faire suite à une catastrophe naturelle.

Peut-on espérer une autre fin?

${ }^{8}$ Mutation : réorganise le système, change sa structure par un saut logique, une discontinuité, une bifurcation. 


\section{RÉFÉRENCES}

CHARREL, M. (2017). Des subprimes à la crise européenne, l'effet domino. Le Monde économie 06.07.2017.

Courrier hebdomadaire $\mathrm{n}^{\circ}$ 13. La grève générale en Belgique. (Décembre 1960-Janvier 1961) - I , Centre de Recherche et d'informations Socio-politique Crisp (1961).

FEAUX, V., (1963). Cinq semaines de lutte sociale. La grève de l'hiver 1960-1961, Éditions de l'Institut de Sociologie - ULB, Bruxelles, 1963.

LE GOLF, J.-J., (2002). Mai 68 : l'héritage impossible 1998. La Découverte, 2002.

PIECQ, A., (2011). De la pensée systémique à la pratique de l'organisation - Le giroscope, L'Harmattan, Paris.

Ouvrage collectif, (1988). Université libre de Bruxelles. Mai 68 : 20 ans déjà.

TOQUEVILLE, A. (1866). L'ancien régime et la révolution livre I - II - III - IV édition : JM. 